\title{
Arbor
}

\section{Los nuevos escenarios para la cooperación en la lucha contra el terrorismo internacional ${ }^{1}$}

\section{Denise Sorasio $^{2}$}

Arbor CLXXII, 678 (Junio 2002), 311-323 pp.

El terrorismo constituye una de las amenazas mas serias para la democracia, para el libre ejercicio de los derechos del hombre y para el desarrollo económico y social.

Los acontecimientos del 11 de septiembre mostraron que el terrorismo es una amenaza real a nivel mundial y que cualquier país podía ser afectado por ataques terroristas. Es un verdadero reto para el mundo y para Europa.

La Unión Europea se fijó como objetivo en el tratado de la UE «ofrecer a los ciudadanos un alto grado de seguridad dentro de un espacio de libertad, seguridad y justicia elaborando una acción en común entre los Estados miembros en los ámbitos de la cooperación policial y judicial en materia penal...».

Este objetivo ha de lograrse mediante la prevención y la lucha contra la delincuencia, organizada o no, en particular el terrorismo a través de una mayor cooperación entre las fuerzas policiales, las autoridades aduaneras y otras autoridades competentes de los Estados miembros, como entre les autoridades judiciales $y$, también, por la aproximación, cuando proceda, de las normas de los Estados miembro en materia penal.

\section{Cooperación judicial penal}

Es muy notable la reacción rápida a nivel de la Unión Europea depués del 11 de septiembre. Este resultado se debe mayormente a la estrecha 
cooperación que se ha desarrollado entre las distintas instituciones europeas, Parlamento Europeo, Consejo y Comisión, facilitando la consecución de acuerdos en breves períodos de tiempo. Todo ello fue posible gracias a que tanto el Parlamento Europeo como la Comisión europea habían estado trabajando durante meses en las medidas para mejorar la lucha contra el terrorismo.

El Parlamento Europeo adoptó el 5 de septiembre de 2001 una recomendación al Consejo ${ }^{3}$ sobre la base de un informe de iniciativa, del que fue ponente el Sr.Watson, relativo al papel de la Unión Europea en la lucha contra el terrorismo. En esta recomendación se resaltaba el profundo cambio del tipo de terrorismo en la Unión Europea, el cual ponía en evidencia la insuficiencia de los métodos clásicos de cooperación judicial y policial para hacerles frente. Este nuevo terrorismo, recalcaba dicha recomendación, es el resultado de la actividad de redes organizadas a escala internacional, establecidas en varios países, que aprovechan los vacíos jurídicos de la territorialidad de la acción judicial y a veces gozan de importante apoyo logístico y financiero. Estas apreciaciones se confirmaron, desgraciadamente, con los acontecimientos del 11 de septiembre en Estados Unidos.

En el Consejo JAI del 20 de septiembre de 2001 (donde la Comisión europea presentó sus propuestas de Decisión marco sobre la definición del delito de terrorismo ${ }^{4}$ y sobre la orden de detención europea adoptadas el día antes ${ }^{5}$ ) y en la reunión extraordinaria del Consejo Europeo el 21 de septiembre de 2001, se alcanzó un compromiso importante: en cuanto a la cooperación judicial, debería lograrse un acuerdo sobre ambos instrumentos y sobre el establecimiento de Eurojust, a más tardar, en el Consejo JAI de 6-7 de diciembre 2001.

Se cumplió este compromiso y en el Consejo de Justicia e Interior del 6-7 de diciembre se alcanzó un acuerdo político, sujeto a algunas reservas parlamentarias y a la reconsulta del Parlamento europeo, sobre la Decisión marco sobre la lucha contra el terrorismo, la cual está destinada a implementar el Artículo 31(e) del Tratado de la Unión Europea, que prevé la adopción progresiva de medidas que establezcan normas mínimas relativas a los elementos constitutivos de los delitos y a las penas en el ámbito, entre otros, del terrorismo. La idea que subyace es que los elementos constitutivos del delito y las sanciones aplicables a una serie de conductas delictivas deben perseguirse con la misma intensidad en todo el territorio de la Unión Europea, ya sea bien por su dimensión transfronteriza o por su especial gravedad.

A pesar de la dificultad derivada del hecho de que sólo siete Estados Miembros tengan regulado como delito específico el delito de terrorismo, 
Los nuevos escenarios para la cooperación...

la Decisión marco contiene una definición de delito terrorista atendiendo a un doble elemento: subjetivo y objetivo.

El elemento subjetivo está configurado por la motivación del terrorista, y para su realización se han tenido en cuenta los criterios establecidos en el Convenio de las Naciones Unidas para la Supresión de la Financiación del Terrorismo (1999) y en la legislación penal de los Estados Miembros. Para estar ante un delito terrorista la motivación debería caer dentro de una de estas tres categorías: o intimidar seriamente a la población, o obligar al Gobierno o a una organización internacional a hacer o a abstenerse de hacer algo, o desestabilizar seriamente o destruir las estructuras fundamentales políticas, constitucionales, económicas o sociales de un país o una organización internacional.

El elemento objetivo viene establecido por una lista de delitos comunes que, cometidos con la especial motivación terrorista, serán calificados como delito terrorista. La pena aplicable a estos será más grave que la establecida para los delitos comunes de la lista cometidos sin la motivación terrorista.

Finalmente, tanto la dirección como la participación en grupos terroristas son también tipificados en esta Decisión marco, siendo castigados con una pena máxima de al menos 15 y 8 años, respectivamente.

Como resultado directo, la Decisión marco facilitará la cooperación policial y judicial, puesto que las definiciones comunes de delitos se requieren para ciertas formas de auxilio judicial. Además, la existencia de un marco común en la lucha contra el terrorismo en la UE facilitará una más estrecha colaboración con terceros países y la puesta en práctica de instrumentos internacionales relacionados con la prevención y supresión del terrorismo, como el convenio de la ONU para la supresión de la financiación del terrorismo previamente mencionado y el futuro convenio sobre el terrorismo internacional.

El Parlamento Europeo ha respondido positivamente a la reconsulta ${ }^{6}$, y una vez que se levanten algunas reservas parlamentarias subsistentes el instrumento será adoptado oficialmente, dejando a los Estados miembros un plazo limite bastante breve para la transposición en su legislación nacional (31 de diciembre 2002).

En relación con la Decisión marco sobre la orden europea de detención, el acuerdo fue algo más difícil de alcanzar, y se logró en las vísperas del Consejo Europeo de Laeken del pasado mes de diciembre.

Con este instrumento se mejora de manera muy importante la cooperación judicial entre los Estados Miembros, de tal modo que los procedimientos de extradición regulados en sus grandes líneas por el Convenio europeo de extradición de 1957, que sometía a la extradición a una serie 
de requisitos tales como la necesidad de doble incriminación, la no extradición de nacionales y la exclusión de los delitos políticos y fiscales que en la práctica constituyen obstáculos insalvables para la extradición-, son definitivamente eliminados y sustituidos por este nuevo sistema. El nuevo instrumento se basa en una decisión judicial dictada en un Estado Miembro, y dirigida a la autoridad de otro Estado Miembro, con el propósito de búsqueda, captura y entrega de la persona, bien sea sospechoso de haber cometido un delito castigado con una pena privativa de libertad de al menos doce meses en el Estado Miembro que solicita la entrega, o bien haya sido condenado a una pena de al menos cuatro meses en el Estado requeriente.

El mecanismo está basado en el reconocimiento mutuo de resoluciones judiciales, que según lo establecido en las conclusiones de Tampere, debe ser la piedra angular de la cooperación judicial tanto en materia civil como penal en el seno de la Unión Europea. El sistema será lo más simple posible: cuando la autoridad de un Estado Miembro solicite que una persona sea detenida, bien en base de una sentencia condenatoria o en el curso de un procedimiento criminal, esa decisión ha de ser reconocida y aplicada en todo el territorio de la Unión Europea. Por otra parte, los motivos para rechazar la ejecución de la petición del Estado requeriente son extremadamente limitados, sin que, por ejemplo, la nacionalidad sea causa que justifique tal rechazo. Cabe destacar la extraordinaria celeridad del proceso, que durará como máximo sesenta días, ampliables a noventa en casos excepcionales.

Para una lista de 32 delitos que deberán estar castigados en el Estado requirente con una pena privativa de libertad de al menos tres años, la entrega de la persona no requerirá el previo control del principio de doble incriminación. Entre estos delitos están no sólo el terrorismo sino la trata de seres humanos, explotación sexual de menores, tráfico ilícito de drogas y de armas, corrupción y fraude, entre otros.

El Parlamento Europeo respondió positivamente a la reconsulta ${ }^{7}$ del Consejo, y una vez que se levanten algunas reservas parlamentarias subsistentes, el instrumento será adoptado oficialmente, dejando a los Estados miembros un plazo limite para la transposición que es el 31 de diciembre de 2003. Algunos Estados miembros han anunciado su voluntad de poner en obra dicha decision-marco en la primera mitad del año 2003.

El Consejo JAI de 28 de febrero llegó a un acuerdo, supeditado a la retirada de diversas reservas de estudio parlamentario y a una nueva consulta al Parlamento Europeo, sobre la propuesta de Decisión marco relativa al reconocimiento mutuo en la Unión europea de las resoluciones de embargo preventivo de bienes y de aseguramiento de pruebas. El progra- 
ma de medidas destinado a poner en práctica el principio del reconocimiento mutuo en materia penal ${ }^{8}$ adoptado por el Consejo el 29 de noviembre de 2000, fijaba como una de las primeras prioridades la adopción de un instrumento que aplicase el principio del reconocimiento mutuo al embargo preventivo de bienes y al aseguramiento de pruebas.

El objetivo del citado instrumento es la ejecución directa y automática por la autoridad competente del llamado «Estado de ejecución» de una resolución de embargo preventivo o de aseguramiento de pruebas transmitida por la autoridad competente del llamado «Estado de emisión», utilizando un formulario único y con arreglo a unas condiciones determinadas.

Dicha resolución, que sería transmitida por la autoridad judicial que la haya dictado, directamente a la autoridad competente para su ejecución, sería ejecutada sin demora del mismo modo que lo sería una resolución de embargo preventivo o de aseguramiento de pruebas nacional.

Por lo que se refiere al ámbito de aplicación de la Decisión marco, será equivalente al acordado para la Decisión marco relativa a la orden de detención europea, es decir, supresión de requisito de la doble incriminación para una lista de 32 delitos, siempre que dichos delitos estén castigados con una pena de al menos tres años. Las resoluciones de embargo preventivo deberán efectuarse respetando los principios de legalidad, subsidiariedad y proporcionalidad.

Dentro de los avances en materia de cooperación judicial hay que referirse a un instrumento que será muy importante en la lucha contra el crimen organizado y el terrorismo, sobre el que hubo también acuerdo político en el Consejo JAI del 6 de diciembre último: y adopción formal el 28 de febrero de 2002, con entrada en vigor el 6 de marzo: Eurojust ${ }^{9}$. Este organismo esta compuesto por fiscales, y magistrados de los Estados miembros y su misión consiste en facilitar la adecuada coordinación de las fiscalías nacionales, así como apoyar las investigaciones penales en los casos de delincuencia organizada y transfronteriza.

La aplicación del principio de reconocimiento mutuo y en general la mejora de los mecanismos de asistencia judicial pasan por la aplicación de una serie de medidas de acompañamiento encaminadas a incrementar el entendimiento de los respectivos sistemas judiciales. En particular, cabe mencionar la Red Judicial Europea ${ }^{10}$, en funcionamiento desde hace más de tres años y que tiene como función facilitar el establecimiento de contactos adecuados entre autoridades judiciales con responsabilidades en el marco de la cooperación judicial internacional, de manera general y para ciertos delitos graves, entre los que se encuentra el terrorismo. 
En fin, la Unión Europea ha actuado en el ámbito del bloqueo de capitales de organizaciones terroristas ${ }^{11}$. Una Posición común del Consejo ${ }^{12}$ fue adoptada el 27 de diciembre de 2001, sobre la aplicación de medidas específicas de lucha contra el terrorismo, para la aplicación de la Resolución 1373 (2001) del Consejo de Seguridad de la ONU sobre el combate al terrorismo. El objetivo de esta acción común era doble: afirmaba como un objetivo de la política exterior y de seguridad común que la Comunidad Europea actúe para asegurar que no se pongan a disposición de las personas, grupos y entidades relacionados en el anexo al texto, ningún fondo o recurso económico ${ }^{13}$. Por otra parte, establecía que los Estados miembros, mediante una cooperación policial y judicial en asuntos penales en el marco del título VI del Tratado de la Unión Europea, se prestarán mutuamente la asistencia más amplia posible para la prevención y la lucha contra actos terroristas.

España ha presentado recientemente un proyecto de decisión relativa a la aplicación de medidas específicas de cooperación policial y judicial en la lucha contra el terrorismo de acuerdo con el Artículo 4 de la Posición Común del Consejo antes mencionada. El objetivo de esta iniciativa es el establecimiento en cada Estado miembro de puntos de contacto que tengan acceso y recopilen información relativa a investigaciones criminales respecto de delitos terroristas en que intervengan cualquiera de las personas, grupos o entidades que figuran en la lista de la Posición común. Según el texto del proyecto, estas informaciones se comunicarían a Europol y Eurojust y se haría pleno uso de los equipos conjuntos de investigación a los efectos de investigar y perseguir los delitos terroristas. De este modo se establecería también un vinculo estrecho entre los instrumentos de cooperación judicial penal y los de cooperación policial.

\section{Cooperación policial}

La acción común en el ámbito de la cooperación policial incluye:

a) La cooperación operativa entre las autoridades competentes, incluidos los servicios de policía, de aduanas y otros servicios especializados en los Estados miembros.

b) La recogida, almacenamiento, tratamiento, análisis e intercambio de información pertinente, en particular mediante Europol.

c) La cooperación y las iniciativas conjuntas en la formación, el intercambio de funcionarios de enlace, el uso de equipos y la investigación científica policial. 
d) La evaluación común de técnicas especiales de investigación relacionada con la detección de formas graves de delincuencia organizada.

El Consejo Europeo de Tampere concluyó que:

«6. Las personas tienen derecho a esperar que la Unión afronte la amenaza que para su libertad y sus derechos civiles constituye la gran delincuencia; Para contrarrestar esta amenaza se precisa un esfuerzo común que prevenga y combata la delincuencia y las organizaciones delictivas en toda la Unión. Es necesaria la movilización conjunta de los recursos policiales y judiciales para garantizar que en toda la Unión no exista lugar alguno donde puedan ocultarse los delincuentes....»

«43. Debe sacarse el máximo provecho de la cooperación entre las autoridades de los Estados miembros al investigar la delincuencia transfronteriza en cualquier Estado miembro. El Consejo Europeo hace un llamamiento para que se creen sin demora equipos conjuntos de investigación tal como se contempla en el Tratado, como primer paso para luchar contra el tráfico de drogas y la trata de seres humanos, así como contra el terrorismo»

«44. El Consejo Europeo pide que se establezca una Unidad operativa europea de jefes de policía con objeto de intercambiar, en cooperación con Europol, experiencia, mejores prácticas e información...»

«45. Europol...su papel debe reforzarse mediante la recepción de datos operativos procedentes de los Estados miembros y la autorización para pedir a los Estados miembros que inicien, dirijan o coordinen investigaciones o creen equipos conjuntos de investigación en determinados ámbitos de la delincuencia...»

Como se ha ya mencionado, el Consejo Europeo extraordinario de 21 de septiembre de 2001 estableció un plan de acción de la Unión Europea como respuesta a los atentados en Estados Unidos e impulsó una amplia gama de medidas legislativas y operativas destinadas a reforzar la cooperación policial y judicial, a contener la financiación del terrorismo, a reforzar la seguridad aérea y a responder a las amenazas de utilización de armas biológicas y químicas.

Concretamente en lo que se refiere a la cooperación policial destacan las siguientes medidas:

- Lista común de organizaciones terroristas internacionales. Dicha lista se elaboró en estrecha cooperación con los servicios operativos responsables de combatir el terrorismo, incluidos los servicios de 
información, las fuerzas de policía y las autoridades judiciales. En relación con esta lista son de aplicación las medidas recogidas en el Reglamento 2580/2001 del Consejo mencionado anterioremente.

- Identificación de los supuestos terroristas en Europa y de las organizaciones que los apoyan a fin de establecer una lista común de organizaciones terroristas autóctonas. Con relación a esta lista impuso la necesidad de una mejor cooperación y un mejor intercambio de información entre todos los servicios de información de la Unión (Art. 4 de la Posición Común 2001/931/PESC).

- Necesidad de acelerar la creación de equipos conjuntos de investigación de la convención relativa a la asistencia judicial en materia penal entre los Estados miembros ${ }^{14}$.

Según el convenio las autoridades competentes de dos Estados miembros pueden crear un equipo común de investigación, con un objetivo preciso y por una duración limitada para efectuar investigaciones penales en uno o varios Estados miembros. Un equipo común de investigación puede crearse cuando:

a) en el contexto de un procedimiento de investigación realizado por un Estado miembro para detectar infracciones, hay que efectuar investigaciones difíciles que implican la movilización de medios importantes que afectan también a otros Estados;

b) varios Estados miembros efectúan investigaciones relativas a infracciones que en razón a los hechos que han dado lugar a su iniciación, exigen una acción coordinada y concertada en los Estados miembros en cuestión.

Respondiendo a los deseos el Consejo varios Estados miembros (Francia, España, Bélgica, Irlanda y el Reino Unido) presentaron una iniciativa para la adopción por el Consejo de una decisión-marco sobre la creación de dichos equipos ${ }^{15}$. La decisión marco publicada el 20.10.2001 estableció que tales equipos deberían crearse con prioridad para luchar contra las infracciones cometidas por los terroristas. Los Estados miembros deben adoptar todas las medidas necesarias para adecuarse a las disposiciones de la Decisión marco antes del 1 de julio de 2002.

- Creación de un equipo de especialistas en antiterrorismo en el seno de Europol. Dicho equipo deberá colaborar estrechamente con sus homólogos estadounidenses. Además el equipo se encarga de mantener actualizado el análisis de la amenaza del terrorismo islámico en Europa. Para permitir la realización de su tarea los Estados miembros fueron exhortados a compartir con Europol, sin demora y sistemáticamente, todo dato útil en materia de terrorismo. Se decidió también otorgar créditos suplementarios, aumer tando el pre- 
supuesto 2002 de Europol de 3,7 M Euro para la lucha contra el terrorismo.

- Refuerzo de la cooperación entre los jefes de las unidades antiterroristas. Para ello se insistió en la necesidad de realizar reuniones periódicas del Grupo Operativo de jefes de Policía.

El Grupo Operativo de jefes de policía realizó tras su reunión de 30/10/01 una serie de recomendaciones para reforzar la cooperación policial en el ámbito de la lucha contra el terrorismo entre las que destacan:

- Sobre el tratamiento de la información:

- Que en el ámbito nacional la evaluación de la amenaza terrorista sea realizada por el servicio competente de manera periódica y sobre la base de criterios comunes.

-Que el colegio de policía CEPOL elabore módulos de formación en el ámbito de la información relativa a la lucha contra el terrorismo.

- Se constituya en Europol un «Centro de Datos sobre artefactos explosivos (Bomb Data Center)».

- Se aligere el procedimiento del Artículo 99 de la Convención de aplicación del Acuerdo Schengen.

- Se busque un mejor equilibrio entre las reglas de protección de datos y las necesidades de las investigaciones concretamente en lo que se refiere a las telecomunicaciones y los datos electrónicos.

- Sobre las medidas para garantizar un nivel elevado de seguridad:

- Incentivar los trabajos para armonizar los aspectos técnicos de la fabricación y la concepción de documentos de identidad, de estancia y de viaje.

- Establecer una mejor cooperación entre los servicios de policía, las compañías aéreas y los servicios de seguridad aérea.

- Sobre la cooperación de las unidades operativas de intervención.

- Creación de una red segura de información y de comunicación entre las unidades operativas antiterroristas.

Mejor cooperación y mejor intercambio de información entre todos los servicios de información e inteligencia.

Para ello deben fomentarse reuniones periódicas de los jefes de los servicios de información.

Refuerzo de la cooperación entre sérvicios de policía, con inclusión de Europol y de los servicios de información.

Este asunto es objeto de discusión para buscar las fórmulas y métodos adecuados de encauzar las relaciones y el intercambio de información entre estos servicios teniendo en cuenta ante todo las especificidades de 


\section{Denise Sorasio}

la organización, estructura, dependencias y competencias de los servicios de información en cada Estado miembro.

Mejora de la coordinación entre Europol/Eurojust y el Grupo Operativo de Jefes de Policía.

El Consejo propuso tras la reunión del 29/10/2001 examinar la compatibilidad de los instrumentos jurídicos de creación de Europol y Eurojust para evitar crear competencias concurrentes y garantizar la complementariedad de las tareas y de los objetivos. Se insistió en la necesidad de establecer mecanismos de información mutua así como favorecer la creación de equipos comunes de investigación como apoyo a la cooperación judicial.

Actualización del repertorio de competencias de Europol.

El ámbito de responsabilidad y las competencias de Europol deben ampliarse a todas las formas de delincuencia citadas en el Convenio y deben ser actualizadas periódicamente para incluir cualquier potencial nueva forma de delincuencia.

Evaluación de los dispositivos nacionales para luchar contra el terrorismo.

Esta tarea será acometida por dos expertos nacionales en aspectos legislativos relacionados con la lucha contra el terrorismo que serán contratados por la Secretaría General del Consejo.

Refuerzo de los controles en las fronteras exteriores y posibilidad de recurso coordinado al apartado 2 del artículo 2 del Convenio Schengen (restablecimiento de los controles en las fronteras) en el caso de amenazas terroristas de extrema gravedad.

Se decidió que constituye una amenaza terrorista de extrema gravedad, a los efectos de aplicación del citado Artículo 2.2 del Convenio:

a. Una amenaza inminente o la ejecución de diversos atentados terroristas simultáneos o sincronizados en varios Estados miembros;

b. Un atentado terrorista de excepcional gravedad cuando existen indicaciones serias de que los autores o los cómplices pueden intentar escapar a otros Estados miembros;

c. Una amenaza inminente de un atentado terrorista de excepcional gravedad en uno o varios Estados miembros cuando existen indicios serios de que los autores o los cómplices pueden proceder de otros Estados miembros.

Refuerzo de las normas de seguridad en los aeropuertos y aviones.

Se ha propuesto un reglamento ${ }^{16}$ que recoge las reglas comunes aplicables en el marco de la seguridad aérea y cuyo principal objetivo es asegurar un nivel elevado de seguridad a través de medidas que impidan los actos ilícitos en la aviación civil. 
Intensificación de la cooperación y armonización de las legislaciones en materia de armas y explosivos.

Diversos trabajos se han acometido en la Comisión para reforzar las medidas de control y vigilancia de la adquisición, el transporte, la comercialización y el consumo de los explosivos de uso civil para evitar que puedan ser sustraídos o desviados para su utilización por organizaciones o elementos terroristas.

\section{Conclusión}

Se ha demostrado que la Unión Europea podía responder de una forma rápida y enérgica a la situación generada después del 11 de septiembre, pero al mismo tiempo que lo podía hacer de forma respetuosa con los derechos fundamentales y las libertades públicas.

Aunque todavía quedan pasos que dar en la consecución de un verdadero espacio de libertad, de seguridad y de justicia, los niveles de cooperación judicial penal que se alcanzarán con la plena aplicación de estos nuevos instrumentos legales garantizarán una coordinación y una rapidez de actuación entre las autoridades judiciales de los Estados Miembros inimaginables hace unos años.

Ninguno de estos instrumentos puede ser juzgado desde el punto de vista de la simple efectividad judicial y policial. Todos estos instrumentos constituyen una garantía de nuestro sistema de libertades común. El terrorismo es un atentado directo contra los derechos y libertades fundamentales reconocidos en los países de la Unión Europea.

Por otra parte la lucha contra el terrorismo exige una acción rotunda y coordinada por parte de las fuerzas de seguridad. La cooperación fluida entre los servicios preventivos y represivos de todos los países afectados es la piedra angular de la eficacia en la lucha contra el terrorismo.

Después del 11 de septiembre se han dado pasos importantes en el ámbito de la cooperación policial pero existen todavía aspectos de esta cooperación que deben ser mejorados. Los esfuerzos deben dirigirse a:

- Hacer esfuerzos para que Europol sea reconocido por todos los servicios policiales como «lugar de encuentro» europeo de la cooperación policial y del intercambio de información.

- La cooperación policial hasta ahora se ha basado en los acuerdos bilaterales. Es necesario ampliar este marco a la participación multinacional. 
- Es necesario progresar en la mejora de la relación permanente y el intercambio entre los servicios de policía y los de inteligencia de los Estados miembros.

Por fin, hay que señalar la intensificación de la cooperación con los Estados Unidos en la lucha contra el terrorismo.

El Consejo de Justicia y Asuntos de Interior (JAI) del 20 de septiembre adoptó una serie de medidas para mejorar la cooperación con los Estados Unidos (EUA) en la lucha contra el terrorismo. La mayor parte de estas medidas tienen que ver con la cooperación judicial y penal. En este ámbito, se identificaron tres áreas específicas de cooperación.

Primero, se invitó a Europol a concluir un acuerdo formal de cooperación con los EUA lo más pronto posible. El 6 de diciembre de 2001 un acuerdo de cooperación estratégica fue firmado por Europol y los EUA. Este acuerdo permite, entre otros, intercambios de información estratégica sobre análisis de tendencias criminales, articulación de mejores prácticas para enfrentar problemas criminales específicos, incluyendo el terrorismo y el intercambio de oficiales de enlace. Actualmente se está negociando un segundo acuerdo incluyendo la posibilidad de intercambiar datos personales. Para que dicho acuerdo pueda ser concluido, Europol necesita convencerse de que los EUA aseguran «un nivel adecuado de protección de datos» para los datos personales transmitidos desde la Unión Europea (UE).

Por otro lado el Grupo operativo de jefes de policía recibió el mandato de la puesta en común con los servicios homólogos de los Estados Unidos de buenas prácticas en el ámbito de la lucha contra en terrorismo.

Segundo, se concedió un papel importante a la nueva agencia Eurojust en el establecimiento de la cooperación judicial. A este fin, magistrados de Eurojust visitaron a sus interlocutores estadounidenses y viceversa, para establecer contacto e informarse unos a otros sobre investigaciones y casos en curso. Además, los EUA nombraron a un oficial de enlace en Eurojust y los Estados miembros de la UE identificaron expertos para facilitar los contactos. Un acuerdo formal de cooperación entre Eurojust y los EUA se negociará en el futuro cercano.

Por último, el Consejo JAI acordó proponer a los EUA negociar un acuerdo de cooperación judicial en materia penal con la UE. En particular, este acuerdo cubriría tanto la extradición como la asistencia legal recíproca. Las primeras pláticas informales entre ambos lados sobre este tema empezaron en febrero de 2002 y continúan en la fecha. Se espera que el Consejo otorgue a su Presidencia, asistida por la Comisión, la autorización para que pueda iniciar formalmente las negociaciones antes del fin de la actual Presidencia española. 


\section{Los nuevos escenarios para la cooperación...}

\section{Notas}

${ }^{1}$ La autora agradece a sus colegas Yolanda Gallego-Casilda y Eduardo Cano Romera por la ayuda proporcionada en la redacción de este artículo.

${ }^{2}$ Este artículo refleja exclusivamente las opiniones de su autor. En ninguna circunstancia estas opiniones deben considerarse la posicíon oficial de la Comisión Europea.

${ }^{3}$ Recomendación del Parlamento Europeo sobre le papel de la Unión en la lucha contra el terrorismo. (A5-0273/2001).

${ }^{4} \mathrm{COM}(2001) 521$ final de 19.9.2001.

5 COM (2001)522 final de 19.9.2001.

6 A5-0003/2002.

7 A2-0003/2002.

8 DOCE C12 de 15.1.2001, p.10.

9 Doce L 63 de 6.3.2002, p. 1.

10 Creada por la Acción común del Consejo de 29 de junio de 1998 (DOCE L191 de 7.7.98, p.4

11 El Consejo adopto también el 27 de diciembre de 2001 otra posición común 2001/930/PESC en la que se recogen varias medidas de lucha contra el apoyo y la financiación del terrorismo entre las que figuran :

- La incriminación especifica de la recolección de fondos para la realización de actos terroristas

- La congelación de los fondos y bienes de los terroristas y de las personas o entidades que actúan en nombre o siguiendo instrucciones.

- La represión del apoyo, el reclutamiento y el aprovisionamiento de armas para los grupos terroristas.

- El rechazo del asilo para los miembros de organizaciones terroristas.

- La asistencia mutua entre los Estados miembros y con los países terceros en las investigaciones contra los actos de terrorismo y de financiación de estas actividades.

12 DOCE L344 de 28.12.2001, p.93.

13 En el ámbito del tratado comunitario esto dio lugar a la adopción del Reglamento (CE)2580/2001 del Consejo de 27 de diciembre de 2001 sobre la adopción de medidas restrictivas especificas contra ciertas personas y entidades en el marco de la lucha contra el terrorismo que permite la congelación de fondos y bienes de los individuos, grupos y entidades recogidos en la lista de la Decisión del Consejo 2001/927/CE.

14 JO C197 12.07.200 Acto del Consejo de 29 de mayo de 2000. Artículo 13.

15 JO C295 du 20.10.2001, (2001/C 295/06).

16 JO C51E du 26.2.2002, COM52001 575 final - 2001/0234(COD). Propuesta de reglamento del Parlamento Europeo y del Consejo relativo a la instauración de reglas comunes en el ámbito fr la seguridad de la aviación civil. 